\title{
Light-Activated Nuclear Translocation of Adeno-Associated Virus Nanoparticles Using Phytochrome B for Enhanced, Tunable and Spatially-Programmable Gene Delivery
}

Eric J. Gomez ${ }^{1}$, Karl Gerhardt ${ }^{1}$, Justin Judd ${ }^{1, \dagger}$, Jeffrey J. Tabor ${ }^{1,2,3}$, Junghae Suh ${ }^{1,2 *}$

${ }^{1}$ Department of Bioengineering, Rice University, Houston, TX

${ }^{2}$ Systems, Synthetic, and Physical Biology Program, Rice University, Houston, TX

${ }^{3}$ Department of Biochemistry and Cell Biology, Rice University, Houston, TX

*Corresponding author

Keywords: optogenetics, phytochrome, light-switchable, light-activatable, stimulus-responsive, viral gene delivery, synthetic biology, synthetic virology 


\section{Nucleotide sequence for PhyB917-His 6}

atgggtgcttcaggtgtatctggtgttggtggttctggtggtggaagaggtggaggtagaggaggtgaagaagaaccatcaagtagtcatacacctaac aatcgtagaggtggtgagcaagctcaatcatcaggtacaaaatcattacgtccaagaagtaatactgaatcaatgtcaaaagcaattcaacaatacacag tagatgctagattacacgccgtattcgaacaatctggagaaagtggtaagagttttgattactcacaatcattgaaaacaaccacttatggtagttcagttcc agaacaacaaatcactgcatatcttagtagaatacaacgtggtggttacattcaaccatttggttgtatgattgcagttgatgaatcttcttttagaatcattgg ttattcagaaaatgcaagagaaatgttgggtatcatgccacaatcagtaccaaccttagaaaaaccagaaattcttgcaatgggtacagatgttagaagttt gtttacatcatcatcatcaattcttttggagagagctttgttgcacgtgaaatcactttacttaatccagtatggattcatagtaagaatactggaaagccattc tatgcaattcttcatagaatagatgtaggagttgttattgatcttgagccagcaagaacagaagatccagcattatctattgctggtgcagtacaatcacaaa aacttgctgttagagcaattagtcaattacaagccttgccaggtggtgatataaaacttctttgtgatacagttgttgaatcagttcgtgatcttaccggttatg atagagttatggtatacaaattccatgaggatgaacatggtgaagttgttgcagaaagtaaaagagatgatcttgaaccatacattggtttgcattatccag ctactgatattccacaagcatcaagatttcttttcaaacaaaatcgtgttagaatgattgtagattgtaatgccacccagtattagttgttcaagatgatagat tgacacaaagtatgtgtttagtaggttcaacattaagagcacctcatggatgtcattcacaatatatggccaatatgggttcaatagcatcattagctatggc agtaatcatcaatggaaatgaagatgatggttcaaatgttgcatcaggtagaagttcaatgcgtttatggggtttagtagtttgtcatcatacaagttctcgtt gtatccatttcctttacgttatgcatgtgaatttcttatgcaagcatttggtttacaattgaatatggaacttcaattagcattacaaatgagtgaaaagagagt tttacgtacacaaacattgttatgcgatatgttattgagagattctccagctggtattgttactcaatcaccatctatcatggatcttgtaaagtgtgatggtgca gcattcttataccacggaaagtactatccattaggtgttgcaccatctgaagttcaaatcaaagatgttgtagaatggttattggctaatcacgcagattctac tggtttatcaactgattctcttggtgatgctggttatcctggtgccgcagccttaggagatgctgtatgtggtatggccgttgcttacattacaaaaagagatt tcttgttttggtttcgttctcatacagctaaagagatcaaatggggtggtgcaaaacatcatccagaagataaggatgatggtcaaagaatgcatccaagat catcatttcaagcattcttagaagtagttaagtcaagaagtcaaccttgggaaacagcagaaatggatgcaatacattcattacaattgatacttcgtgattc attcaaagaatcagaagcagcaatgaatagtaaagttgttgatggtgttgttcaaccatgtagagatatggccggtgaacaaggtattgatgaattaggtg ctgtagctagagaaatggttagattgatagaaactgccactgttccaatcttcgctgttgatgctggtggatgcataaacggttggaatgctaagatcgca gaattgaccggtttgtcagttgaagaagctatgggtaaaagtttagtttcagatttgatctataaggaaaatgaagcaaccgttaacaaattgttatcaagag cattgagaggagatgaggaaaagaatgtagaagttaagttaaagacattttcaccagagttacaaggtaaagcagtttttgttgtagttaatgcttgttcatc aaaagattacttgaataacattgtaggtgtttgttttgttggtcaagatgtaacttcacaaaagattgttatggataagtttatcaatatccaaggtgattacaaa gctattgttcattctccaaatccattgattccaccaatctttgcagctgatgagaatacatgttgtttagaatggaatatggcaatggaaaagttaactggttg gtcacgttcagaagtaattggtaagatgattgttggagaggtttttggtagttgttgtatgcttaaaggtccagatgctttaactaagtttatgattgttttgcata atgcaattggtggtcaagatacagataagttcccattccctttcttcgatagaaatggaaagtttgttcaagcattacttactgctaacaaaagagtatcatta gaaggtaaagtaataggagctttttgtttcttacaaattccttcaccagaattacaacaagctcttgcagtaggtggtagtcatcatcatcatcatcattaa

\section{Refer to citations for constructs already published by others.}


a

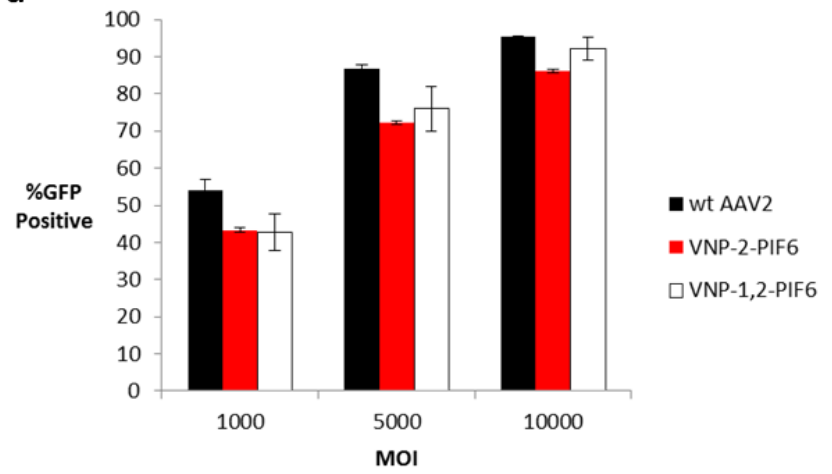

b

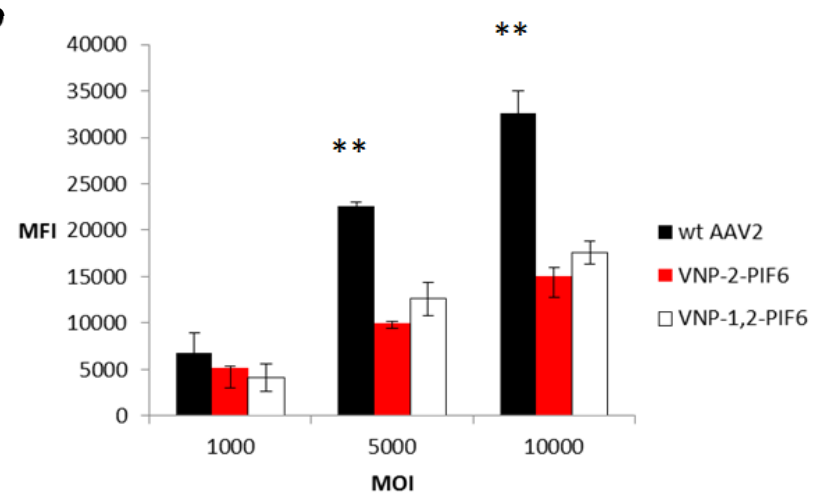

Supplementary Figure 1. VNP-PIF6 is defective in transduction. (a) \% GFP positive cells quantified by flow cytometry on HEK293T cells transduced with wt AAV2, VNP-2-PIF6, or VNP-1,2-PIF6 at MOIs 1,000, 5,000, and 10,000. (b) Mean fluorescence intensity (MFI) quantified by flow cytometry on HEK293T cells transduced with wt AAV2, VNP-2-PIF6, or VNP-1,2-PIF6 at MOIs 1,000, 5,000, and 10,000. wt exhibits significantly higher MFI compared to virions harboring PIF6 insertions. Asterisks indicate $P$-value $<0.05$ by unpaired Student's $t$-test. Error bars are SEM from 4 (wt and VNP-2-PIF6) or 2 (VNP-1,2-PIF6) independent experiments conducted in duplicate. 

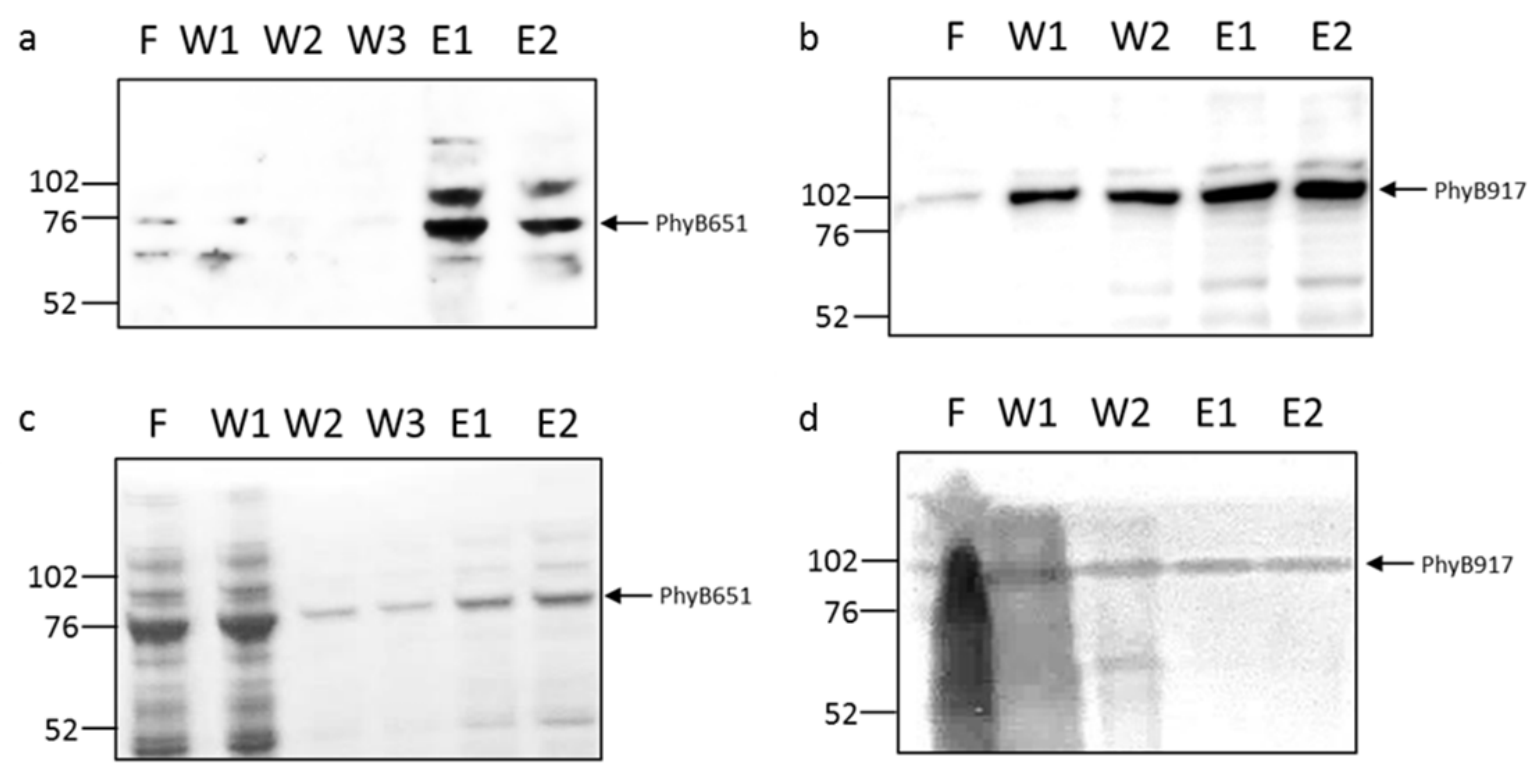

Supplementary Figure 2. PhyB651 and PhyB917 can be purified in vitro. (a, b) Western blots probed with anti-PhyB antibody for PhyB651 (a) and PhyB917 (b) showing $\mathrm{Ni}^{2+}$ fractions from left to right ( $\mathrm{F}=$ flow through, W1=first wash, W2=second wash, W3=third wash, E1=first elute, E2=second elute). PhyB bands can be observed predominantly in elute fractions, with PhyB651 appearing at 76kDa, and PhyB917 appearing at $102 \mathrm{kDa}$ ladder marks. (c, d) Coomassie stains showing purified $\mathrm{Ni}^{2+}$ fractions from left to right ( $\mathrm{F}=$ flow through, W1=first wash, W2=second wash, W3=third wash, E1=first elute, E2=second elute) for PhyB651 (c) and PhyB917 (d). Elute fractions indicate mostly pure samples. 
a

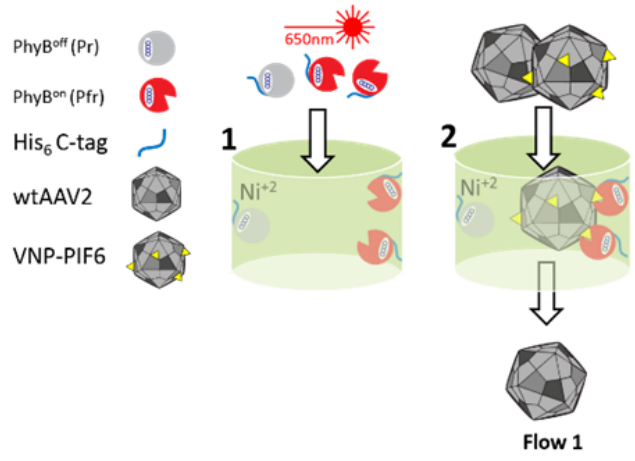

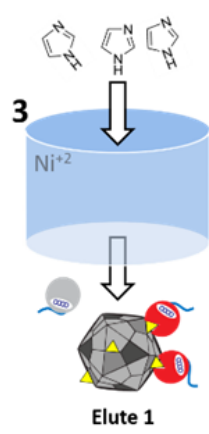

4

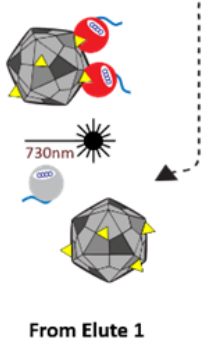

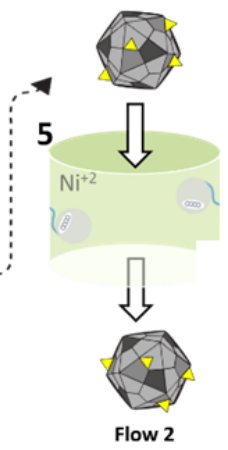

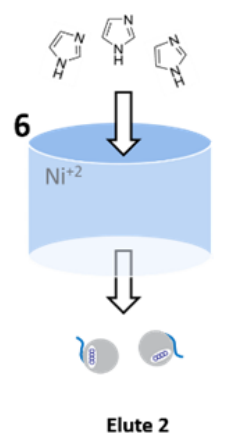

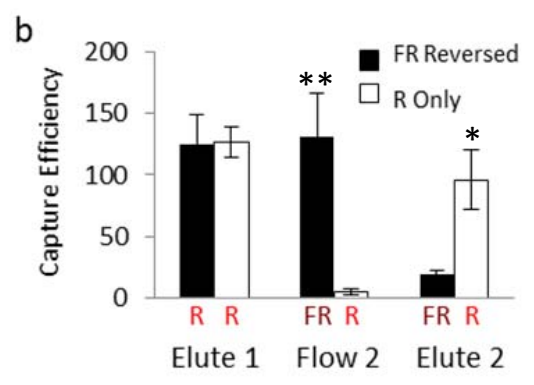

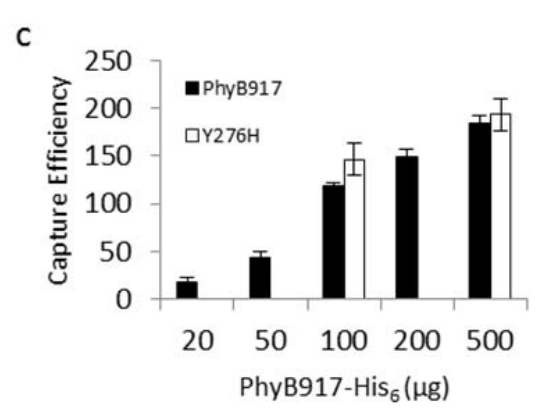

Supplementary Figure 3. VNP-PIF6 binding to PhyB917 in vitro can be reversed with FR light. (a, b) To probe for deactivation of PhyB917 using FR light in vitro, $\mathrm{Ni}^{2+}$ column elute fractions containing activated PhyB917 bound to VNP-PIF6 (i.e. Elute 1) were diluted to $20 \mathrm{mM}$ imidazole and exposed to FR light for 20 min (step 4). The FR-treated samples were then applied to a new $\mathrm{Ni}^{2+}$ column, and new flow (step 5, Flow 2) and elute (step 6, Elute 2) fractions were collected. After inactivation with FR light in step 4, the majority of VNP-PIF6 was detected in the flow through (Flow 2), and not in the following elute fraction (Elute 2), indicating VNP-PIF6 binding to PhyB917 is reversible with FR light exposure (asterisks indicate $P$-value $<0.001$ by unpaired Student's $t$-test). In contrast, the control sample of Elute 1 treated with R light at step 4 resulted in majority of viruses being bound to the column in step 5 and eluting in step 6 (Elute 2, asterisks indicate $P$-value $<0.005$ by unpaired Student's $t$-test). Error bars are

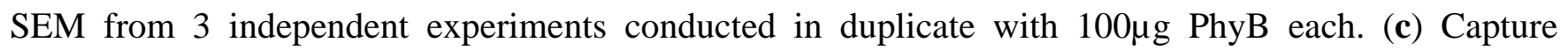
efficiency for regular PhyB917 versus constitutively active PhyB917(Y276H) mutant. To verify that the observed light-dependent binding is a function of the phytochrome and not some other nonspecific mechanism, the constitutively active PhyB917(Y276H) mutant was tested alongside PhyB917. The capture efficiency between PhyB917 and PhyB917(Y276H) was statistically non-significant at the two PhyB concentrations tested ( $P$-value $=0.09$ and 0.376 for $100 \mu$ g and $500 \mu$ g data points). Error bars are SEM from 2 independent experiments conducted in duplicate. 

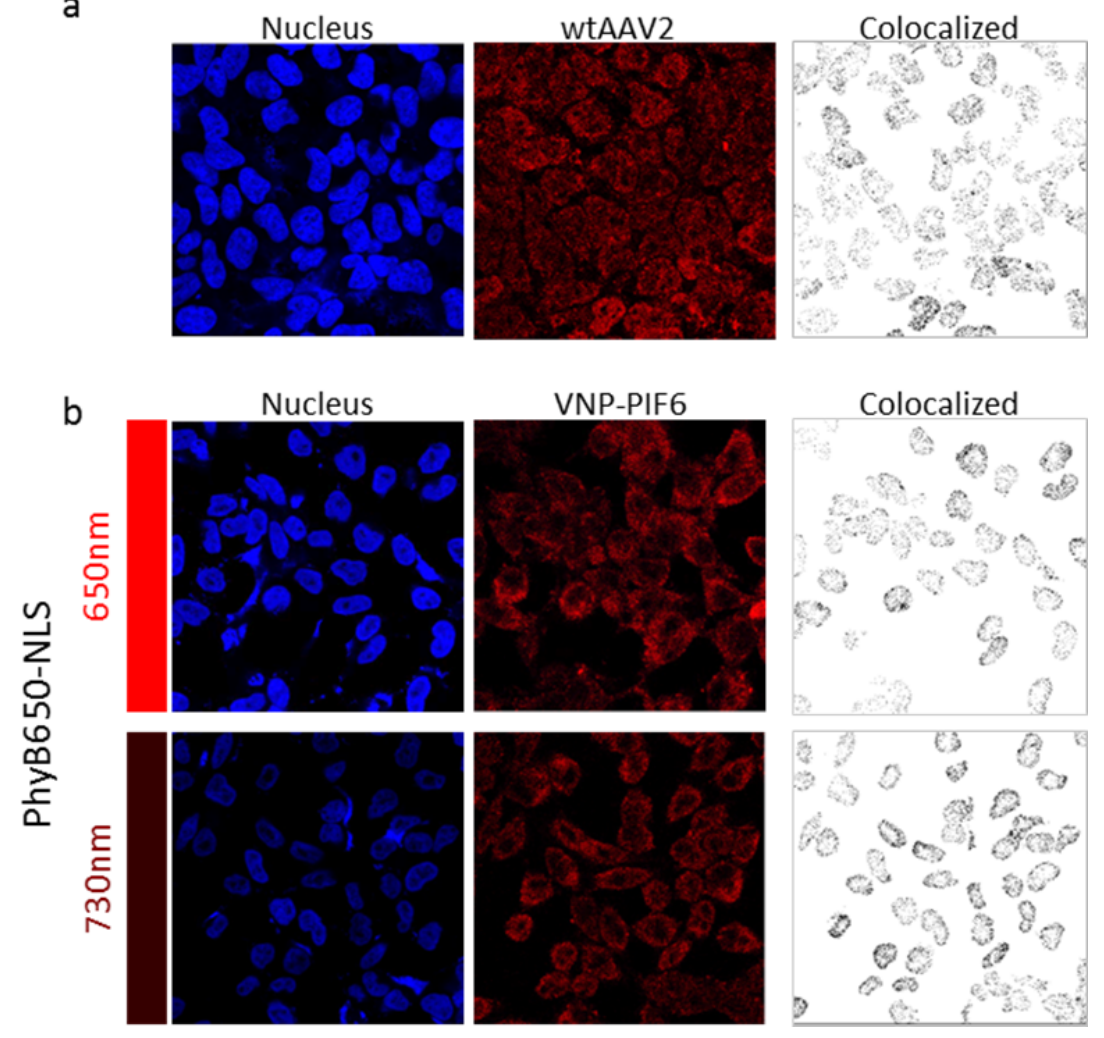

Supplementary Figure 4. wt AAV2 and VNP-PIF6 in the presence of PhyB650-NLS do not exhibit increased nuclear localization. Confocal images showing nuclear and A20 signals for (a) wt AAV2, and (b) VNP-PIF6 (MOI: 5,000) in cells expressing PhyB650-NLS exposed to R or FR light. Left panel: Hoechst nucleus stain. Middle panel: A20 anti-AAV full-capsid antibody. Right panel: object-based colocalized pixels. Object-based colocalization was used to segment nuclei and qualitatively determine overlap with A20 signal. 
$24 \mathrm{~h}$ post-transduction
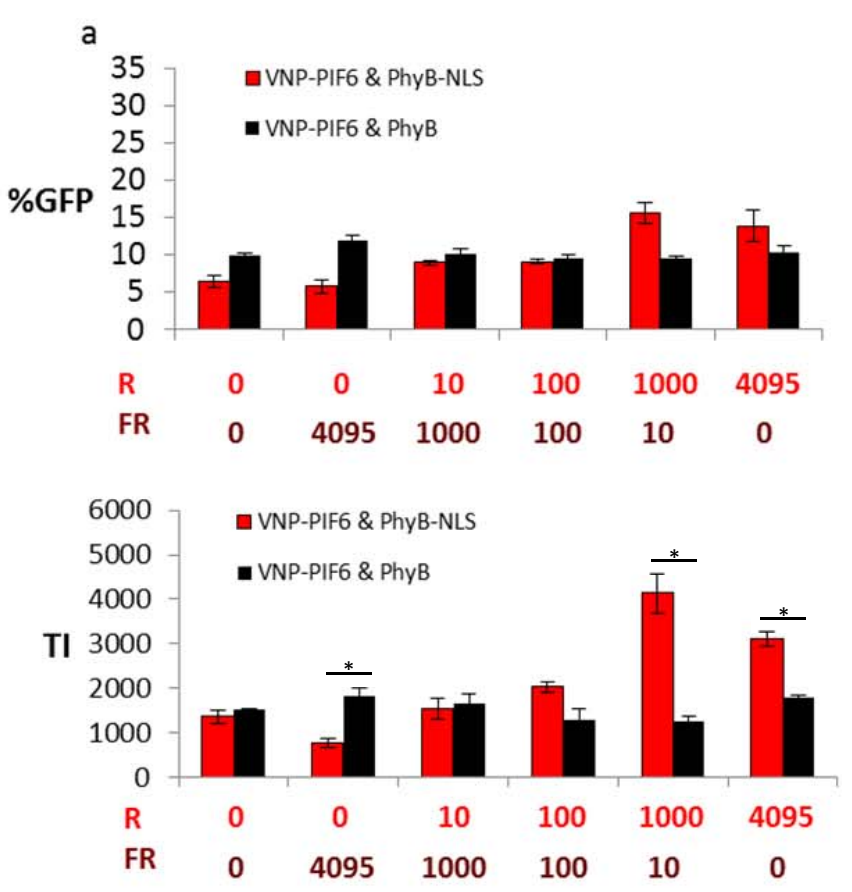

$48 \mathrm{~h}$ post-transduction

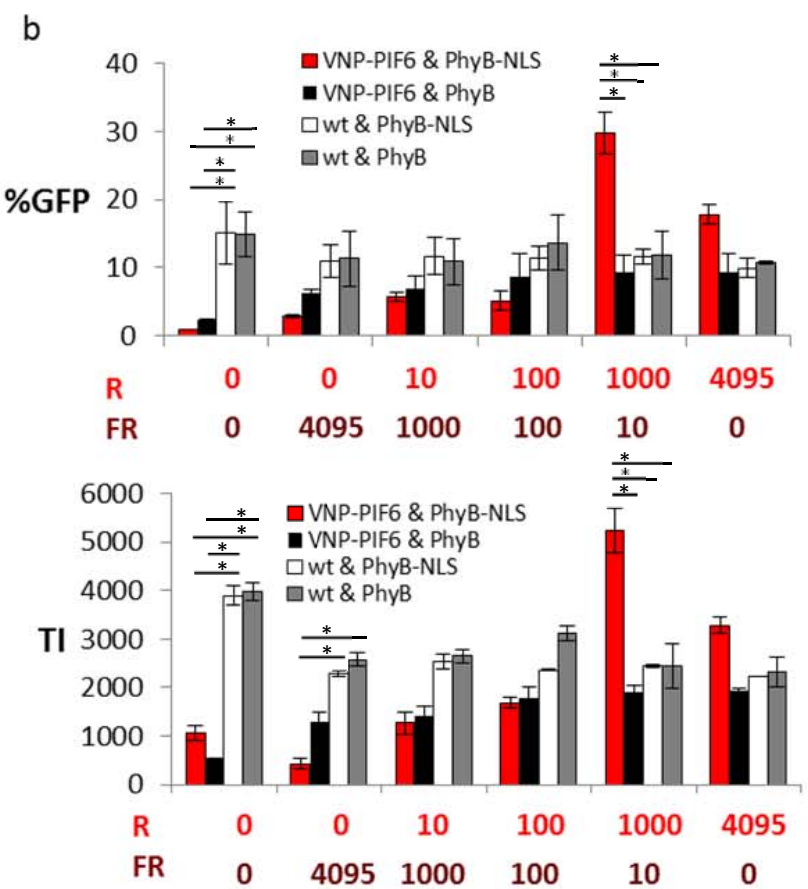

Supplementary Figure 5. Gene expression by VNP-PIF6 is induced by $R$ light in cells expressing PhyB-NLS. (a) \% GFP ${ }^{+}$cells and transduction index (TI) of HeLa cells expressing PhyB or PhyB-NLS transduced by VNP-PIF6, quantified 24h post-transduction. Cells were exposed to different intensities of $\mathrm{R}$ and FR light shown on the $\mathrm{x}$-axis. Asterisks indicate significant difference between PhyB and PhyBNLS $\left(P\right.$-value $<0.05$ ) by unpaired Student's $t$-test. (b) Same as in (a), except $\% \mathrm{GFP}^{+}$cells and TI data are reported for $48 \mathrm{~h}$ post-transduction and wt AAV2 samples are included. wt AAV2 is relatively unaffected by the presence of PhyB or by exposure to light. Asterisks indicate significant difference between groups $(P$-value $<0.05)$ by unpaired Student's $t$-test. Error bars are SEM from 2 independent experiments conducted in duplicate. 
a

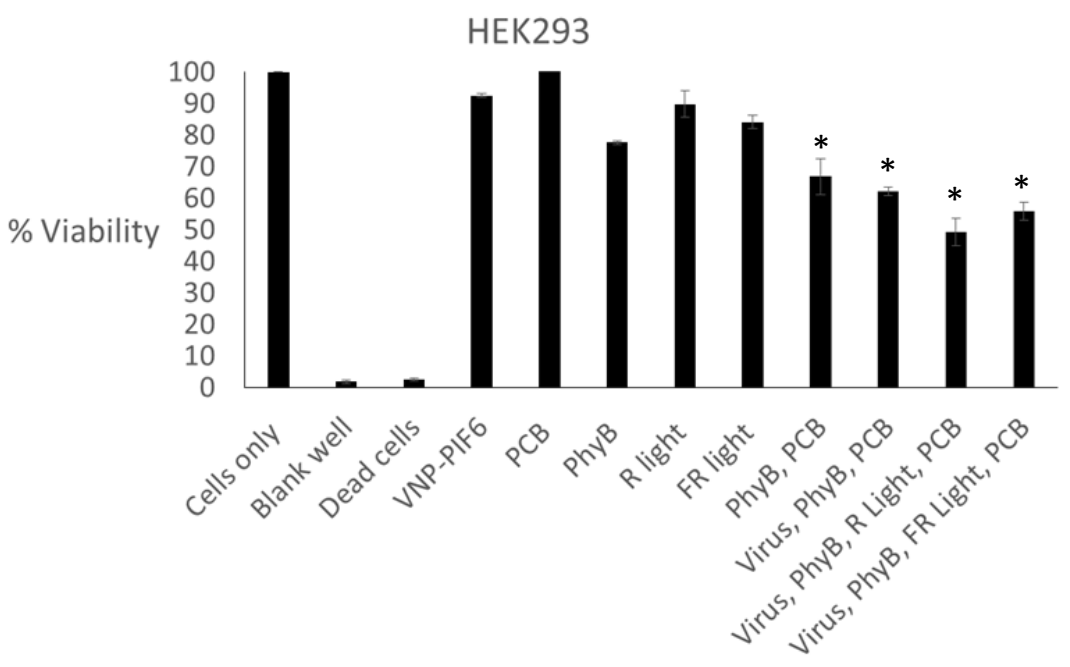

b

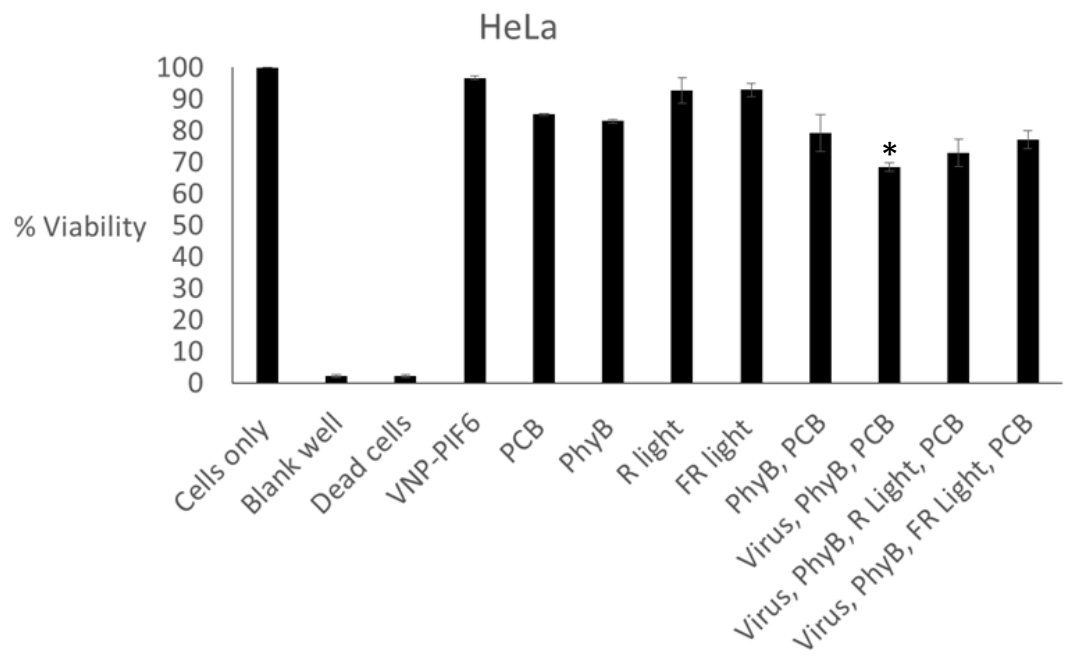

Supplementary Figure 6. Cell metabolic activity assay indicates moderate toxicity induced collectively by VNP-PIF6, PhyB, PCB, and light. Viability of (a) HEK293T or (b) HeLa cells 48h after transduction by VNP-PIF6 (MOI: 2,000), transfection with PEI:PhyB-NLS, addition of PCB $(15 \mu \mathrm{M})$, and/or exposure to $\mathrm{R}$ light $\left(10.67 \mu \mathrm{mol} / \mathrm{m}^{2} \mathrm{~s}\right)$ or FR light $\left(3.61 \mu \mathrm{mol} / \mathrm{m}^{2} \mathrm{~s}\right)$, as evaluated by an MTT assay measuring cell metabolic activity (performed according to manufacturer's protocol). Dead cells control samples were treated with $1 \%$ Triton X-100 for 1min. PEI-mediated transfection of PhyBNLS may be the most toxic single factor. The combination of virus, PhyB-NLS, and PCB (with or without R light, depending on the cell type) results in the greatest toxicity overall. Asterisks indicate significant difference compared to cell only control $(P$-value $<0.005)$ by unpaired Student's $t$-test. 


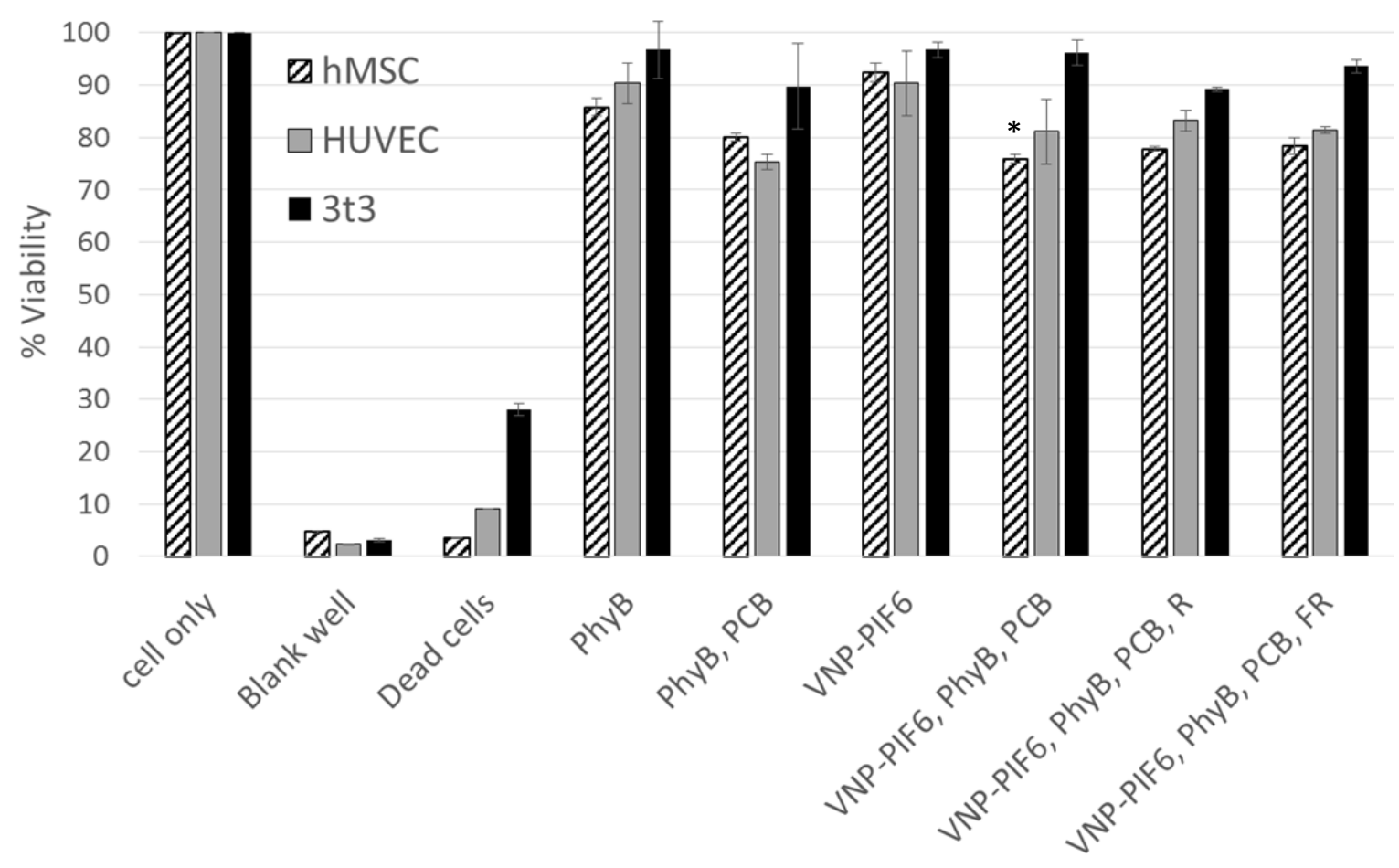

Supplementary Figure 7. Cell metabolic activity assay for additional cell lines. Viability of hMSC, HUVEC, or 3T3 cells 48h after transduction by VNP-PIF6 (MOI: 2,000), transfection with PEI:PhyBNLS, addition of PCB $(15 \mu \mathrm{M})$, and/or exposure to R light $\left(10.67 \mu \mathrm{mol} / \mathrm{m}^{2} \mathrm{~s}\right)$ or FR light $(3.61$ $\mu \mathrm{mol} / \mathrm{m}^{2} \mathrm{~s}$ ), as evaluated by an MTT assay measuring cell metabolic activity (performed according to manufacturer's protocol). Dead cells control samples were treated with $1 \%$ Triton X-100 for 1 min. Asterisk indicates significant difference compared to cell only control $(P$-value $<0.005)$ by unpaired Student's $t$-test. 

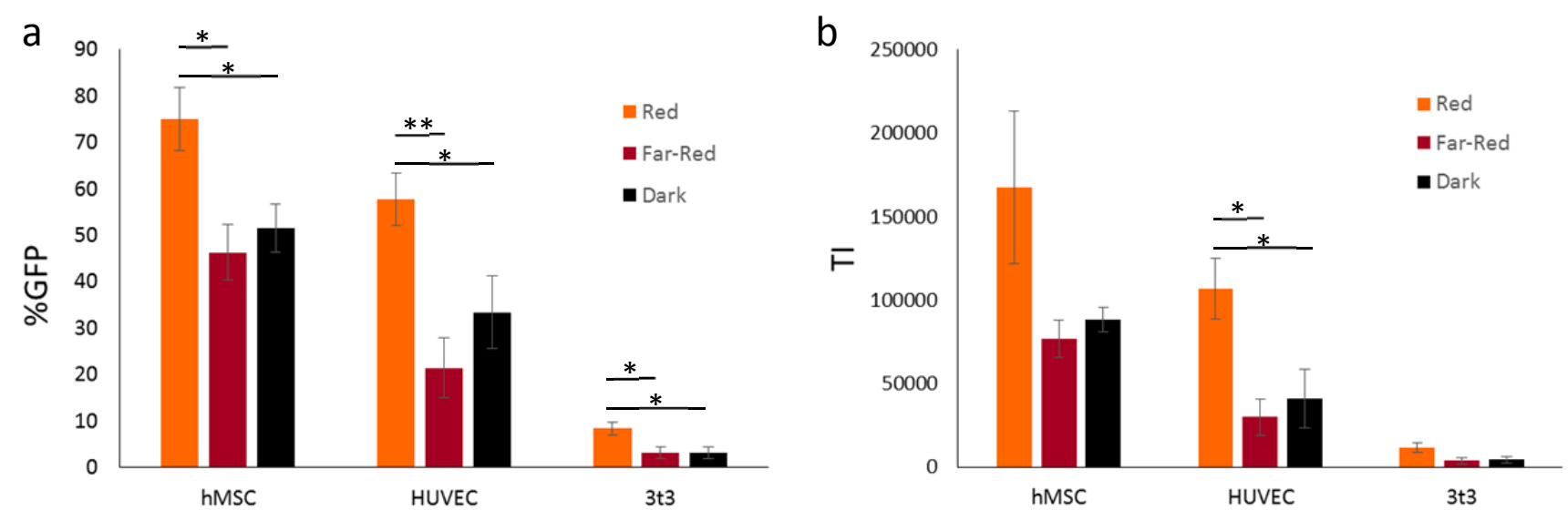

Supplementary Figure 8. Light-activatable viral gene delivery in three other cell lines. Human mesenchymal stem cells (hMSC), human umbilical vein endothelial cells (HUVEC), or 3T3 fibroblasts were seeded in a 24-well plate with opaque walls and lid. Well bottoms are glass and were coated by poly-l-lysine to facilitate cell adherence before transfection with PEI:PhyB-NLS, addition of PCB, and transduction by VNP-PIF6 at an MOI of 2,000. LEDs installed directly underneath each well illuminated R light $\left(10.67 \mu \mathrm{mol} / \mathrm{m}^{2} \mathrm{~s}\right)$ or FR light $\left(3.61 \mu \mathrm{mol} / \mathrm{m}^{2} \mathrm{~s}\right)$ for $48 \mathrm{~h}$ before cells were evaluated by flow cytometry. (a) is the \%GFP positive cells, and (b) is the transduction index (TI, product of the \%GFP+ cells and the mean fluorescence intensity for each group). Compared to control wells not exposed to light, wells exposed to R light transduced at least 2-fold more effectively in all three cell lines, whereas wells exposed to FR light transduced less effectively. The extent that the three cell types are permissive toVNP-PIF6 transduction is variant in that upon R light activation, the achieved TI values are 167,399 for hMSC, 106,866 for HUVEC, and 10,524 for 3T3. Therefore, even in a cell line that is not highly permissive to AAV transduction (i.e. 3T3), we are still able to observe light-activated increase in transduction. Asterisks indicate significant difference of $\mathrm{R}$ light groups compared to others $\left({ }^{*} P\right.$-value $<$ 0.05 , ** $P$-value $<0.005)$ by unpaired Student's $t$-test. 
a

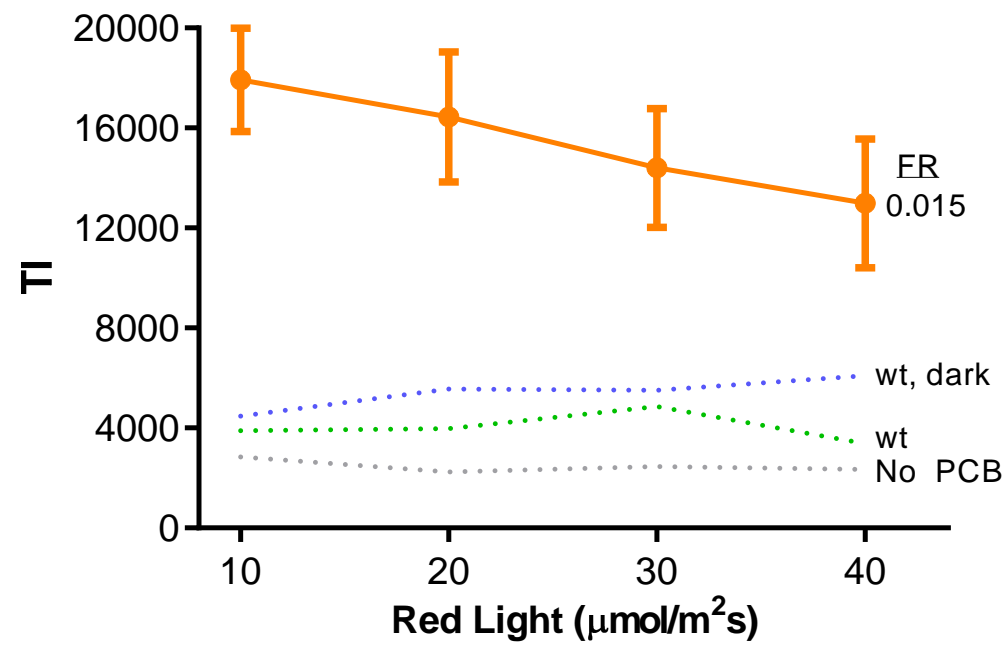

b

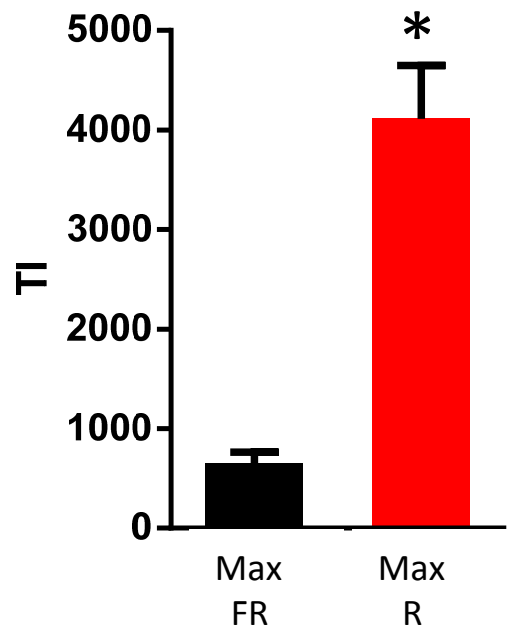

Supplementary Figure 9. Gene delivery by VNP-PIF6 begins to decline at high R light flux. (a) Transfer function for transduction by VNP-PIF6 under low FR $\left(0.015 \mu \mathrm{mol} / \mathrm{m}^{2} \mathrm{~s}\right)$ and increasing $\mathrm{R}$ light flux between 10 and $40 \mu \mathrm{mol} / \mathrm{m}^{2} \mathrm{~s}$. (b) Transduction index for VNP-PIF6 at maximum FR only (15 $\left.\mu \mathrm{mol} / \mathrm{m}^{2} \mathrm{~s}\right)$ and maximum R only $\left(43 \mu \mathrm{mol} / \mathrm{m}^{2} \mathrm{~s}\right)$. Asterisk indicates significant difference $(P$-value $<$ 0.005 ) by unpaired Student's $t$-test. 


\begin{tabular}{cccccc}
\hline Cell line & Virus & Light & tM(nuc) & tM(virus) & PCC \\
\hline hMSC & wt & - & 0.32 & 0.78 & 0.64 \\
hMSC & VNP-2-PIF6 & FR & 0.30 & 0.55 & 0.49 \\
hMSC & VNP-2-PIF6 & $\mathrm{R}$ & 0.51 & $0.97^{*}$ & $0.75^{*}$ \\
\hline HUVEC & wt & - & 0.19 & 0.23 & 0.37 \\
HUVEC & VNP-2-PIF6 & FR & 0.04 & 0.12 & 0.28 \\
HUVEC & VNP-2-PIF6 & $\mathrm{R}$ & 0.15 & 0.39 & $0.45^{*}$ \\
\hline 3t3 & wt & - & 0.17 & 0.43 & 0.34 \\
$3 t 3$ & VNP-2-PIF6 & FR & 0.13 & 0.45 & 0.35 \\
$3 t 3$ & VNP-2-PIF6 & $\mathrm{R}$ & $0.48^{*}$ & $0.72^{*}$ & $0.56^{*}$ \\
\hline
\end{tabular}

Supplementary Table 1. Virus-nucleus colocalization statistics for additional cell lines. Manders' thresholded colocalization and Pearson correlation coefficient (PCC) for hMSC, HUVEC, and 3t3 cells infected with wt AAV2 or VNP-PIF6 (MOI: 5,000). Measurements were determined over three fields of view for each sample, with an average of 24 cells per field of view. tM(nuc) is the proportion of all nuclear signal overlapped by virus signal. tM(virus) is the proportion of all virus signal overlapped by nuclear signal. Nuclear and AAV signals were uniformly thresholded using the ImageJ JACoP plugin. ${ }^{*} P$-value $<0.05$ for $\mathrm{R}$ light condition as determined by two-way ANOVA and Tukey's multiple comparisons test. 